\title{
The Effect of World Trade Center Exposure on the Timing of Diagnoses of Obstructive Airway Disease, Chronic Rhinosinusitis, and Gastroesophageal Reflux Disease
}

OPEN ACCESS

Edited by:

André F. S. Amaral, Imperial College London, UK

Reviewed by:

Sara De Matteis,

Imperial College London, UK

Wan C. Tan,

University of British Columbia,

Canada

*Correspondence:

Charles B. Hall

charles.hall@einstein.yu.edu

Specialty section:

This article was submitted to Epidemiology,

a section of the journal

Frontiers in Public Health

Received: 16 August 2016 Accepted: 16 January 2017 Published: 08 February 2017

Citation:

Liu X, Yip J, Zeig-Owens R, Weakley J, Webber MP,

Schwartz TM, Prezant DJ, Weiden MD and Hall CB (2017) The

Effect of World Trade Center Exposure on the Timing of Diagnoses of Obstructive Airway Disease,

Chronic Rhinosinusitis, and Gastroesophageal Reflux Disease.

Front. Public Health 5:2.

doi: 10.3389/fpubh.2017.00002

\begin{abstract}
Xiaoxue Liu' ${ }^{1,2}$, Jennifer Yip ${ }^{1,2}$, Rachel Zeig-Owens ${ }^{1,2,3}$, Jessica Weakley, Mayris P. Webber, ${ }^{2,3}$, Theresa M. Schwartz ${ }^{1,2}$, David J. Prezant ${ }^{1,2}$, Michael D. Weiden $^{2,4}$ and Charles B. Hall ${ }^{3 *}$

${ }^{1}$ Department of Medicine, Montefiore Medical Center, Bronx, NY, USA, ${ }^{2}$ Bureau of Health Services, Fire Department of the City of New York, Brooklyn, NY, USA, ${ }^{3}$ Department of Epidemiology and Population Health, Albert Einstein College of Medicine, Bronx, NY, USA, ${ }^{4}$ Department of Medicine, New York University School of Medicine, New York, NY, USA
\end{abstract}

Objectives: In a cohort of rescue/recovery workers exposed to the dust that resulted from the collapse of the World Trade Center (WTC), we assessed how a diagnosis of obstructive airways disease (OAD) affected the likelihood of a subsequent diagnosis of chronic rhinosinusitis (CRS) or gastroesophageal reflux disease (GERD). We also assessed whether OAD acted as a mediator of the association between exposure to the WTC rescue/recovery effort and CRS and GERD diagnoses.

Methods: In this prospective cohort study, we analyzed Fire Department of the City of New York physician diagnoses of OAD, CRS, and GERD that were first documented between September 11, 2001, and September 10, 2011, among 8,968 WTC-exposed firefighters. We used piecewise exponential survival models to evaluate whether OAD was a risk factor for either CRS or GERD and to assess OAD as a possible mediator.

Results: An OAD diagnosis significantly increased the risks for subsequent CRS [relative rate $(\mathrm{RR}), 4.24 ; 95 \% \mathrm{Cl}, 3.78-4.76$ ] and GERD (RR, 3.21; 95\% Cl, 2.93-3.52) diagnoses. Further, $21 \%$ of the WTC exposure effect (high vs. low intensity) on GERD and 13\% of the effect (high vs. low intensity) on CRS were mediated by a prior OAD diagnosis.

Conclusion: Individuals with an OAD diagnosis had elevated risks for subsequent diagnoses of CRS or GERD. Part of the effect of WTC exposure on CRS and GERD diagnoses is mediated by prior diagnoses of $O A D$; this mediation effect of $O A D$ may reflect biological pathways or healthcare utilization practices.

Keywords: 9/11, firefighters, piecewise survival model, aerodigestive, obstructive airways disease

\section{INTRODUCTION}

The destruction of the World Trade Center (WTC) buildings in New York City after the terrorist attack on September 11, 2001 resulted in a massive dust cloud containing partially combusted and/or pulverized wood, paper, and jet fuel; pulverized construction materials including asbestos, glass, silica, fiberglass, and concrete; complex organic chemicals; lead and other metals; and 
other potentially hazardous materials (1). Adverse respiratory effects of WTC exposure have been widely documented and have shown consistent dose-response relationships $(2,3)$. We and others have found a high postexposure health burden of aerodigestive conditions, a category that includes lower respiratory diseases such as asthma, chronic bronchitis, and COPD/ emphysema, which together are categorized as obstructive airways disease (OAD); upper respiratory diseases that were predominantly chronic rhinosinusitis (CRS); and gastroesophageal reflux disease (GERD) $(2,4,5)$. In the decade and a half since the disaster, a subgroup of WTC-exposed workers have experienced chronic inflammation at mucosal surfaces in the nose, sinuses, and lungs, producing $\operatorname{CRS}(3,6)$, reactive airway disease, and GERD, which may be due to caustic esophageal exposure in the context of accidental ingestion. The common pathway for these conditions may be postexposure airway inflammation and hyperresponsiveness (7). By 2015, nearly $30 \%$ of Fire Department of the City of New York (FDNY) WTC rescue/recovery workers had a physician diagnosis of CRS, $28 \%$ GERD, and 24\% OAD (8).

High comorbidity rates of WTC-related aerodigestive conditions have been commonly reported (9-12). Notably, the percentage of WTC rescue/recovery workers diagnosed with all three aerodigestive conditions ranged from approximately 10-30\% $(8,10,11)$. Clinical reasons for disease co-occurrence remain unknown. GERD may cause lower and upper respiratory diseases or exacerbate persistent airway irritation $(13,14)$. Alternatively, OAD or CRS may cause or exacerbate GERD through mechanically induced inflammation (e.g., cough, postnasal drip, mucous), drug effects (theophylline, corticosteroids), or shared neurological pathways.

We have previously reported that FDNY WTC rescue/ recovery workers were more frequently diagnosed with $\mathrm{OAD}$ than CRS in the months and years after exposure, but that over time, diagnoses of CRS increased $(2,3,15)$. Similarly, about 6 years postexposure, GERD diagnoses, which had the lowest immediate post-9/11 incidence of these aerodigestive conditions, started to increase (unpublished data). The role, if any, of an early OAD diagnosis in subsequent diagnoses of CRS and/ or GERD was unclear. Given our access to FDNY's electronic medical records system, which includes dates of diagnoses, we explored the associations between post-WTC exposure OAD diagnoses in relation to CRS and GERD in a cohort of FDNY WTC-exposed firefighters. Specifically, our aims were to 1) assess how a diagnosis of OAD affects the likelihood of a subsequent diagnosis of CRS or GERD and 2) assess whether OAD acts as a mediator of the associations between WTC exposure and CRS and WTC exposure and GERD.

\section{MATERIALS AND METHODS}

The source population consisted of FDNY male firefighters who were active (i.e., non-retired) on September 11, 2001 and who arrived at the WTC site to participate in the rescue/recovery effort on or before September 24, 2001; who gave informed consent for research; and who had at least one visit to the FDNY Bureau of Health Services (FDNY-BHS) for treatment of any medical condition after exposure $(N=10,181)$. After excluding firefighters with pre-WTC exposure evidence of OAD, CRS, or GERD in their FDNY-BHS medical records, the final analysis cohort consisted of 8,968 participants.

Demographic information such as age, race, and retirement status was obtained from the FDNY employee database. WTC exposure intensity was obtained from participants' first post9/11 health questionnaire. Since 1996, FDNY-BHS has used an electronic medical record system, which contains in-house physician diagnoses and information from diagnostic tests such as endoscopy, spirometry, methacholine challenge tests, and chest CT scans.

WTC exposure intensity was categorized by time of initial arrival to the WTC site: on the morning of September 11, 2001 (high); in the afternoon of September 11, 2001, or anytime on September 12, 2001 (moderate); and on any day between September 13, 2001, and September 24, 2001 (low) (2, 3, 15).

New-onset OAD, CRS, and GERD conditions were diagnosed by FDNY-BHS physicians. We reviewed the FDNY electronic medical record database for the first documented mention of these conditions between September 11, 2001, and September 10,2011 . We used the same case definitions for OAD and CRS as previously described $(2,3,15)$. Briefly, an OAD case had any of the following: 1) two or more diagnoses of asthma documented at least 30 days apart, 2) two or more diagnoses of COPD/ emphysema documented at least 30 days apart, or 3) two chronic bronchitis diagnoses recorded within 1 year of each other, followed by at least one additional chronic bronchitis diagnosis within the next 3 years. A CRS case required a diagnosis of either CRS or irritant chronic rhinitis and at least one abnormal diagnostic test result (either nasal laryngoscopy or sinus CT scan). A GERD case required two or more diagnoses documented at least 30 days apart of gastroesophagitis, esophagitis reflux, or Barrett's esophagus.

\section{Statistical Analysis}

We separately fit piecewise exponential survival models (15) to assess whether an OAD diagnosis increased the likelihood of a subsequent diagnosis of either CRS or GERD. Piecewise exponential survival models are similar to Cox regression models, but unlike Cox regression models in which the baseline hazard changes with every event, these models allow the baseline hazard to change at a large, but finite number of time points. Because Poisson regression models are mathematically identical to exponential survival models, we used a Poisson likelihood to fit the following models:

$$
\begin{aligned}
& \log \left\{E\left(Y_{i} \mid a_{1 i}, a_{2 i}, m_{i}, c_{i}\right)\right\}=\log \left\{T_{i}\right\}+\alpha_{0}^{*}+\alpha_{1}^{*} a_{1 i}+\alpha_{2}^{*} a_{2 i}+\alpha_{c}^{*} c_{i} . \\
& \log \left\{E\left(Y_{i} \mid a_{1 i}, a_{2 i}, m_{i}, c_{i}\right)\right\}=\log \left\{T_{i}\right\}+\alpha_{0}^{* *}+\alpha_{m}^{* *} m_{i}+\alpha_{c}^{* * \prime} c_{i} . \\
& \log \left\{E\left(Y_{i} \mid a_{1 i}, a_{2 i}, m_{i}, c_{i}\right)\right\}=\log \left\{T_{i}\right\}+\alpha_{0}+\alpha_{1} a_{1 i}+\alpha_{2} a_{2 i} \\
& +\alpha_{m} m_{i}+\alpha_{c}^{\prime} c_{i} \text {. }
\end{aligned}
$$

Here, $Y_{i}$ is the number of incident cases of the outcome of interest (CRS or GERD) for stratum $i$ defined by unique combinations 
of predictors-age as of September 11, 2001, retirement status (time dependent), last known smoking status (never, former, current), and follow-up time (3-month long-time intervals); $T_{i}$ is the total follow-up time at risk across all study participants contributing follow-up time to stratum $i ; a_{1 i}$ is a dummy variable taking the value of 1 for high exposure and 0 for moderate or low exposure; $a_{2 i}$ is a dummy variable taking the value of 1 for moderate exposure and 0 for high or low exposure; $m_{i}$, the mediator, is a time-dependent variable taking the value 1 for strata including follow-up time after individuals have received OAD diagnoses and 0 otherwise; and $c_{i}$ is a vector containing all other covariate values for that stratum. In our analyses, we allowed the baseline hazard to change every 3 months, and dummy variables that accomplish this are included in the vector $c_{i} . Y_{i}$ is modeled as a Poisson random variable and $m_{i}$ as a binary random variable, and a full likelihood approach is used. In addition, in piecewise exponential survival models, actual baseline rates can be estimated from the data, and the relative hazards are also relative rates (RRs). Follow-up time began on September 11, 2001, and ended with the earliest of the following: incident GERD or CRS diagnosis date (depending on the outcome of interest), death date, date of latest treatment visit to FDNY-BHS, or the end of the study period (September 10, 2011). Date of latest FDNY treatment visit was used in follow-up time calculation to ensure that participants had an opportunity to receive an aerodigestive diagnosis. We then included diagnoses of $\mathrm{OAD}$ as a time-dependent predictor to the models to see how it was associated with CRS and GERD. SAS PROC GENMOD was used to fit the models.

Mediation, as schematically depicted by Figure 1, is sometimes measured by the difference in effects between a model that includes the mediator such as Eq. 3 and a model that does not include the mediator such as Eq. 1 above. Relevant effects are defined as

$$
\begin{aligned}
& \mathrm{RDE}_{1}=\alpha_{1}, \\
& \mathrm{TE}_{1}=\alpha_{1}^{*},
\end{aligned}
$$

where $\mathrm{RDE}_{1}=\alpha_{1}$ is the regression direct effect, the log relative hazard (or RR) of incident CRS or GERD from being in the highvs. low-exposure group when OAD is included in the model and $T E_{1}=\alpha_{1}^{*}$ is the total effect, the log relative hazard (or RR) of

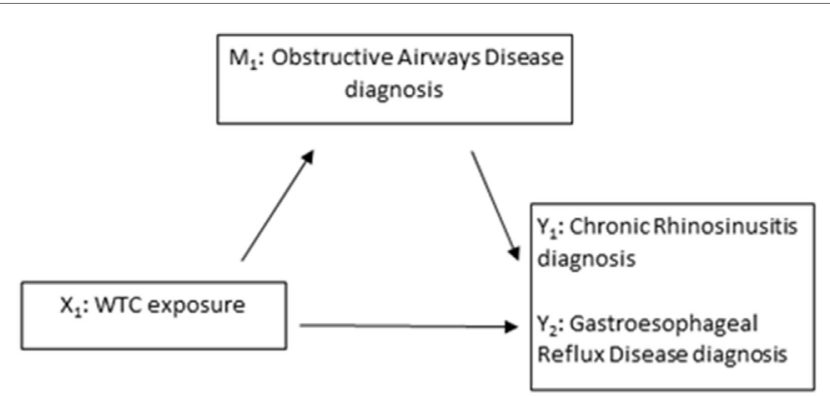

FIGURE 1 | Model schematic of assessing obstructive airways disease as a mediator $\left(M_{1}\right)$ of the association between World Trade Center (WTC) exposure $\left(X_{1}\right)$ and CRS $\left(Y_{1}\right)$ and between WTC exposure and gastroesophageal reflux disease $\left(Y_{2}\right)$. incidence CRS or GERD from being in the high- vs. low-exposure group when OAD is not included in the model. Similar measures are defined for the contrast between moderate- and low-exposure groups. $\alpha_{1}-\alpha_{1}^{*}$ has often been used as a measure of mediation by $m$, but in order for it to be an accurate measure of mediation, the following assumptions must be met: no interaction between the risk factor of interest (here, WTC exposure), no unmeasured confounding, and collapsible measures of effect. The first two assumptions are in doubt in our study, so we also performed a formal causal mediation analysis as follows.

\section{Causal Mediation Analysis}

To better evaluate the possibility that diagnoses of OAD acted as a mediator for the effect of WTC exposure on the incidence of CRS and GERD, we then fit the following model, similar to that of VanderWeele (16):

$$
\begin{gathered}
\log \left\{E\left(Y_{i} \mid a_{1 i}, a_{2 i}, m_{i}, c_{i}\right)\right\}=\log \left\{T_{i}\right\}+\theta_{0}+\theta_{1} a_{1 i}+\theta_{2} a_{2 i}+\theta_{m} m_{i} \\
+\theta_{\text {int } 1} a_{1 i} m_{i}+\theta_{\text {int } 2} a_{2 i} m_{i}+\theta_{c}^{\prime} c_{i} . \\
\log \left\{\frac{\operatorname{Pr}\left(m_{i}=1 \mid a_{1 i}, a_{2 i}, c_{i}\right)}{1-\operatorname{Pr}\left(m_{i}=1 \mid a_{1 i}, a_{2 i}, c_{i}\right)}\right\}=\beta_{0}+\beta_{1} a_{1 i}+\beta_{2} a_{2 i}+\beta_{c}^{\prime} c_{i} .
\end{gathered}
$$

Variables are as defined above for Eqs 1-3. SAS PROC HPNLMOD was used for the model fitting. This approach allowed us to directly estimate the following quantities:

$$
\begin{aligned}
& \mathrm{CDE}_{1}=\theta_{1} . \\
& \mathrm{INT}_{\text {ref } 1}=\theta_{\text {int } 1}\left(\beta_{0}+\beta_{c}^{\prime} c_{i}\right) a_{1 i} . \\
& \mathrm{INT}_{\text {med } 1}=\theta_{\text {int } 1} \beta_{1} . \\
& \mathrm{PIE}_{1}=\theta_{M} \beta_{1} . \\
& \mathrm{TE}_{1}=\mathrm{CDE}_{1}+\mathrm{INT}_{\text {ref } 1}+\mathrm{INT}_{\text {med } 1}+\mathrm{PIE}_{1} .
\end{aligned}
$$

The controlled direct effect $\mathrm{CDE}_{1}=\theta_{1}$ is the effect of the risk factor (WTC exposure-being in the high- vs. the lowexposure group) on the outcome (CRS or GERD diagnosis incidence) when the mediator (OAD diagnosis) is not present; $\mathrm{INT}_{\text {ref } 1}=\theta_{\text {int } 1}\left(\beta_{0}+\beta_{c}^{\prime} c_{i}\right) a_{1 i}$ is the reference interaction between WTC exposure and predictors other than diagnosis of OAD, a measure of the degree of effect modification other than through the mediator; $\mathrm{INT}_{\text {med } 1}=\theta_{\text {int } 1} \beta_{1}$ is the interaction between WTC exposure and the mediator (OAD diagnosis) itself; $\mathrm{PIE}_{1}=\theta_{M} \beta_{1}$ is the pure indirect effect from the product approach; and $\mathrm{TE}_{1}$ is the total effect of exposure on incidence. The proportion mediated is $\mathrm{PIE}_{1} / \mathrm{TE}_{1}$ and is of primary interest. As $\mathrm{INT}_{\text {refl }}$ depends on the additional model predictors; for our analyses, we used the predictors for the last time interval for a never smoking firefighter aged 40-45 years as of September 11, 2001. Similar quantities were estimated for the effect of being in the moderate-vs. low-exposure group. Approximate confidence intervals were estimated using 1,000 bootstrap replications.

Because the FDNY WTC Health Program started to offer free medications for WTC-related health conditions starting in 2007, we performed sensitivity analyses by refitting all models described above, but beginning follow-up time on September 11, 2006. All analyses were conducted in SAS (SAS v9.4, SAS Institute, Cary, North Carolina). 
This study was approved by the Institutional Review Board of the Albert Einstein College of Medicine and Montefiore Medical Center; all study participants gave written consent for research.

\section{RESULTS}

Table 1 shows characteristics of the study population. Most study participants $(71 \%)$ were in the moderate-exposure group, and over a third (37\%) was ever smokers. GERD had the highest post-9/11 incidence (27\%), followed by OAD (23\%) and CRS (19\%). About $96 \%$ of those with post-9/11 GERD had at least one endoscopy. Among those with incident OAD, $87 \%$ had at least one of the following pulmonary assessments: pulmonary functions testing with bronchodilator response, methacholine challenge tests, or chest CT scans. Among those with incident CRS, 100\% had either an abnormal sinus CT or ENT laryngoscopy as at least one abnormal diagnostic test was required in our case definition. There was a clear exposure response gradient for incidences of all three aerodigestive conditions.

Figure 2 shows the crude (unadjusted) incidence rates of OAD, CRS, and GERD by WTC exposure intensity over 10 years of follow-up. Immediately after 9/11, OAD incidence rates were high, whereas the rates of CRS and GERD remained low. Starting in year 6 (2007), rates of all three aerodigestive conditions increased.

In piecewise exponential survival models, individuals with an $\mathrm{OAD}$ diagnosis have elevated risks for subsequent diagnoses of either CRS [RR, 4.24 (95\% CI, 3.78-4.76)] and/or GERD [RR, 3.21 (95\% CI, 2.93-3.52); Table 2]. An OAD diagnosis remains a significant risk factor for both health outcomes after accounting for WTC exposure. Table 2 shows results from Eqs. 1, 2, and 3 above; they suggest a potential role of OAD as a mediator of the association between WTC exposure and CRS and GERD, respectively. Specifically, WTC exposure is significantly associated with both CRS and GERD. After including OAD in each model, the association between WTC exposure and each aerodigestive condition was reduced.

Table 3 shows the results from the causal mediation analysis, Eqs. 5 and 6 above. The estimates for the controlled direct effect are slightly lower than that of the regression direct effects from the models presented in Table 2: For example, in Eq. 3, the log RR (log hazard ratio) for incidence of CRS in the high- vs. low-exposure group was 0.53 vs. 0.49 in the in the causal mediation model 5 and 6. This was also true for the moderate vs. low exposure contrast ( $\log$ RRs 0.34 vs. 0.30 ) and for the corresponding contrasts in the models for GERD incidence ( 0.26 vs. 0.24 for high vs. low exposure and 0.15 vs. 0.12 for moderate vs. low exposure). Interactions are all small and non-significant, and there is some mediation of the effect of WTC exposure on incidence of CRS and GERD by OAD diagnosis: $10-13 \%$ for CRS incidence and $21 \%$ for GERD incidence.

In sensitivity analyses, we found that the estimates from models in which follow-up started on September 11, 2006, were similar to estimates from models displayed in Table 2 (Table 4). For most contrasts, the proportion mediated was lower than for the complete follow-up (Table 5).

\section{DISCUSSION}

We found that WTC-exposed firefighters with an OAD diagnosis had more than four times the risk of subsequently being diagnosed with CRS and three times the risk of being subsequently diagnosed with GERD. Further, we showed that an OAD diagnosis partially mediates the association between WTC exposure and GERD and between WTC exposure and CRS. There are at least two explanations of our findings: biologic, meaning vulnerability of individuals with $\mathrm{OAD}$; and structural, meaning healthcare practices of physicians at FDNY and elsewhere.

In causal mediation analyses, we found that $\mathrm{OAD}$ diagnoses mediated $13 \%$ of the effect of being in the high vs. low WTC exposure group on the incidence of CRS and $21 \%$ of the effect on the incidence of GERD. This is somewhat less than the amount of mediation that would have been estimated by comparing the regression coefficients in models with and without OAD (17 and $34 \%$, respectively). This shows that non-significant interactions, as observed in this study, can affect estimates from the causal mediation analyses. Future studies on mediation should consider the possible effects of interactions on mediation estimates.

Possible biologic explanations include the noteworthy hypothesis that OAD, CRS, and GERD are all a consequence of non-resolving inflammation at aerodigestive mucosal surfaces exposed to caustic WTC dust. Thus, the association of OAD with CRS and with GERD could demonstrate elevated individual risk for mucosal injury due to specific exposure conditions or patient intrinsic vulnerability (3). Another explanation posits that OAD or CRS may cause or exacerbate GERD through mechanically induced inflammation (e.g., cough, postnasal drip, mucous). Third, WTC-exposed individuals with a diagnosis of OAD have diminished capacity for physical activity, possibly leading to reduced fitness and substantial weight gain, which is

TABLE 1 | Characteristics of the Fire Department of the City of New York firefighter study population by World Trade Center (WTC) exposure intensity.

\begin{tabular}{|c|c|c|c|c|}
\hline & $\begin{array}{l}\text { High WTC exposure } \\
\qquad(N=1,473)\end{array}$ & $\begin{array}{c}\text { Moderate WTC } \\
\text { exposure }(N=6,391)\end{array}$ & $\begin{array}{l}\text { Low WTC exposure } \\
\qquad(N=1,104)\end{array}$ & Total $(N=8,968)$ \\
\hline Age on September 11, 2001 (years), median (IQR) & $39.50(34.60-45.30)$ & $39.30(34.20-44.90)$ & $40.90(35.00-46.50)$ & $39.60(34.40-45.10)$ \\
\hline Ever smoker, $N(\%)$ & $546(37.07)$ & $2,358(36.90)$ & $442(40.04)$ & $3,346(37.31)$ \\
\hline Physician visits, median (IQR) & $28(14-47)$ & $28(14-46)$ & $26(11-44)$ & $28(13-46)$ \\
\hline Incident gastroesophageal reflux disease (GERD), N (\%) & $462(31.36)$ & $1,708(26.73)$ & $254(23.01)$ & $2,424(27.03)$ \\
\hline Overall obstructive airways disease incidence per 100 person-years & 3.56 & 2.62 & 1.98 & 2.68 \\
\hline Overall chronic rhinosinusitis incidence per 100 person-years & 2.79 & 2.23 & 1.47 & 2.22 \\
\hline Overall GERD incidence per 100 person-years & 3.49 & 2.92 & 2.48 & 2.96 \\
\hline
\end{tabular}




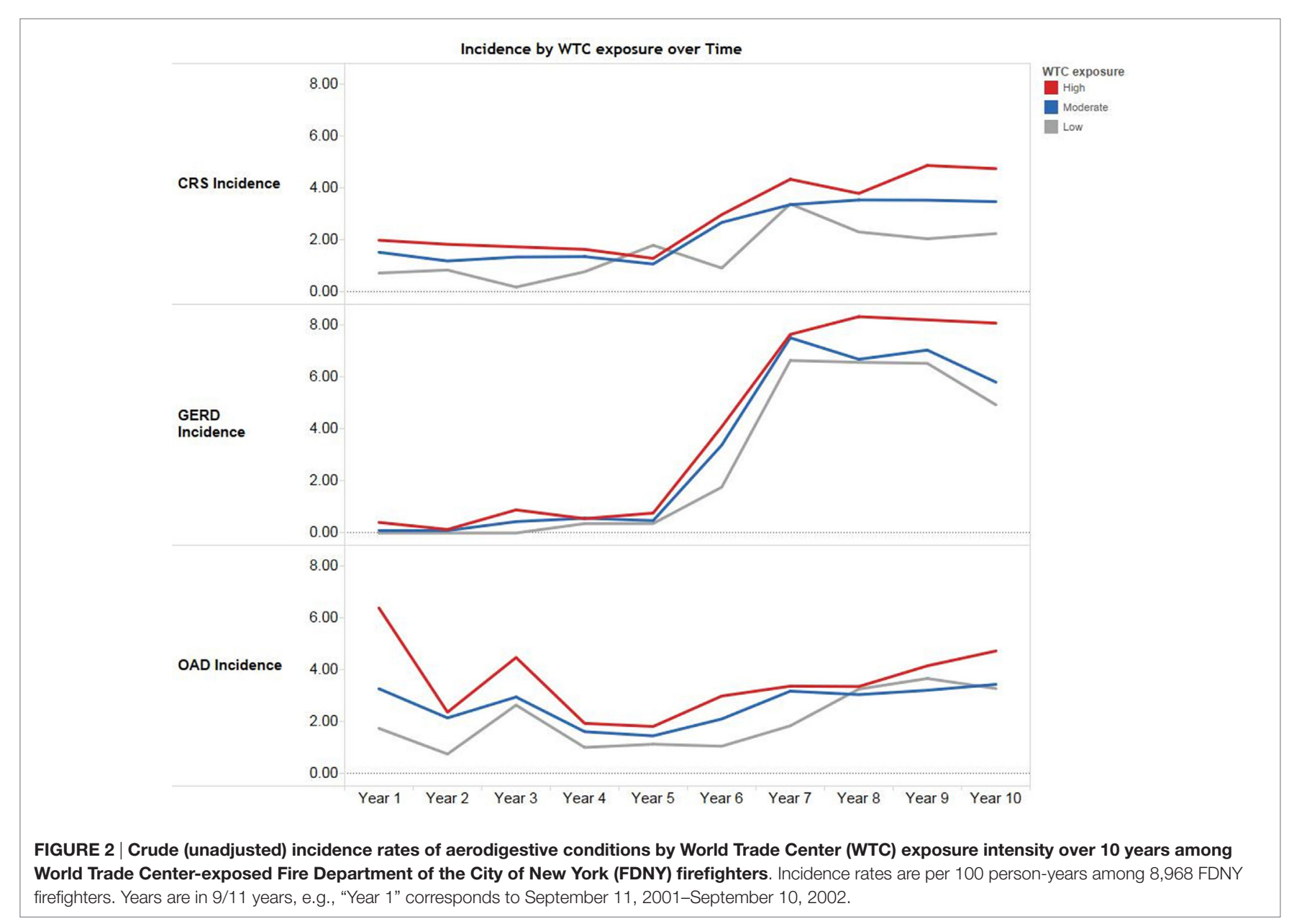

TABLE 2 | Piecewise exponential survival models for the association of WTC exposure, and OAD diagnosis, with CRS and GERD, separately.

\begin{tabular}{|c|c|c|c|c|c|c|}
\hline \multirow[t]{2}{*}{ Estimate } & \multicolumn{2}{|c|}{$\begin{array}{c}\text { Model 1-total effect of WTC } \\
\text { exposure OAD } \\
\text { ignored }\end{array}$} & \multicolumn{2}{|c|}{$\begin{array}{l}\text { Model 2-effect of OAD } \\
\text { diagnosis, WTC exposure } \\
\text { ignored }\end{array}$} & \multicolumn{2}{|c|}{$\begin{array}{l}\text { Model 3-regression } \\
\text { direct effect of WTC } \\
\text { exposure }\end{array}$} \\
\hline & Relative rate & $95 \% \mathrm{Cl}$ & Relative rate & $95 \% \mathrm{Cl}$ & Relative rate & $95 \% \mathrm{Cl}$ \\
\hline \multicolumn{7}{|c|}{ Models for incidence of chronic rhinosinusitis } \\
\hline WTC exposure: high vs. low & 1.90 & $1.56-2.31$ & $\mathrm{~N} / \mathrm{A}$ & & 1.70 & $1.40-2.06$ \\
\hline WTC exposure: high vs. moderate & 1.28 & $1.14-1.44$ & $\mathrm{~N} / \mathrm{A}$ & & 1.21 & $1.08-1.36$ \\
\hline \multicolumn{7}{|c|}{ Models for incidence of gastroesophageal reflux disease } \\
\hline WTC exposure: high vs. low & 1.48 & $1.27-1.73$ & $\mathrm{~N} / \mathrm{A}$ & & 1.30 & $1.11-1.51$ \\
\hline WTC exposure: high vs. moderate & 1.19 & $1.07-1.32$ & $\mathrm{~N} / \mathrm{A}$ & & 1.12 & $1.01-1.24$ \\
\hline WTC exposure: moderate vs. low & 1.25 & $1.09-1.42$ & $\mathrm{~N} / \mathrm{A}$ & & 1.16 & $1.01-1.32$ \\
\hline Effect of OAD diagnosis & $\mathrm{N} / \mathrm{A}$ & & 3.21 & 2.93-3.52 & 3.16 & $2.89-3.47$ \\
\hline
\end{tabular}

All models were fit using piecewise exponential survival models and included age, retirement status, smoking status, and season as covariates. Models are defined in the text in Eqs 1-3.

an independent risk factor for GERD. Finally, OAD medications such as corticosteroids or theophylline may also lead directly to GERD. Non-biologic or structural explanations include the likelihood that individuals with an OAD diagnosis are regularly seen for treatment at FDNY-BHS, which increases the opportunity for those individuals to receive additional diagnoses, including CRS or GERD. At this point, deeper investigation is warranted. The reduced mediation in the sensitivity analyses of the post-2006 
TABLE 3 | Results from causal mediation analyses examining the effect of OAD diagnosis as a mediator for chronic rhinosinusitis and gastroesophageal reflux disease.

\begin{tabular}{|c|c|c|c|c|c|c|}
\hline & \multicolumn{3}{|c|}{ High vs. low exposure } & \multicolumn{3}{|c|}{ Moderate vs. low exposure } \\
\hline & Estimate & \multicolumn{2}{|c|}{$95 \% \mathrm{Cl}$} & Estimate & \multicolumn{2}{|c|}{$95 \% \mathrm{Cl}$} \\
\hline \multicolumn{7}{|c|}{ Causal mediation models for chronic rhinosinusitis diagnosis } \\
\hline Controlled direct effect & 0.485 & 0.300 & 0.632 & 0.302 & 0.085 & 0.495 \\
\hline Interaction in absence of mediator & 0.011 & -0.016 & 0.042 & 0.010 & -0.007 & 0.042 \\
\hline Mediated interaction & 0.011 & -0.013 & 0.038 & 0.005 & -0.003 & 0.017 \\
\hline Pure indirect effect & 0.061 & 0.026 & 0.093 & 0.028 & 0.012 & 0.050 \\
\hline Total effect & 0.567 & 0.377 & 0.718 & 0.344 & 0.137 & 0.539 \\
\hline Proportion mediated & 0.131 & 0.081 & 0.190 & 0.104 & 0.044 & 0.208 \\
\hline \multicolumn{7}{|c|}{ Results from conventional models for chronic rhinosinusitis diagnosis } \\
\hline Regression direct effect & 0.531 & 0.336 & 0.723 & 0.336 & 0.166 & 0.507 \\
\hline Total regression effect & 0.642 & 0.445 & 0.837 & 0.392 & 0.223 & 0.565 \\
\hline \multicolumn{7}{|c|}{ Causal mediation models for gastroesophageal reflex disease diagnosis } \\
\hline Controlled direct effect & 0.243 & 0.073 & 0.427 & 0.124 & -0.024 & 0.274 \\
\hline Interaction in absence of mediator & 0.002 & -0.014 & 0.023 & 0.003 & -0.012 & 0.023 \\
\hline Mediated interaction & 0.002 & -0.020 & 0.025 & 0.002 & -0.008 & 0.011 \\
\hline Pure indirect effect & 0.063 & 0.036 & 0.095 & 0.031 & 0.015 & 0.051 \\
\hline Total effect & 0.310 & 0.146 & 0.486 & 0.160 & 0.023 & 0.302 \\
\hline Proportion mediated & 0.211 & 0.112 & 0.467 & 0.206 & 0.082 & 0.967 \\
\hline \multicolumn{7}{|c|}{ Results from conventional models for gastroesophageal reflux disease diagnosis } \\
\hline Regression direct effect & 0.262 & 0.104 & 0.412 & 0.148 & 0.010 & 0.278 \\
\hline Total regression effect & 0.392 & 0.239 & 0.548 & 0.223 & 0.086 & 0.351 \\
\hline
\end{tabular}

Causal mediation models are defined in Eqs 5 and 6 in the text; conventional models are defined in Eqs 1 and 3 . The quantities shown in the tables are defined in Eqs 7 and 4 in the text. Confidence intervals for causal mediation models based on 1000 bootstrap simulations; confidence intervals for conventional models based are large sample Wald-type intervals.

TABLE 4 | Models for incidence of chronic rhinosinusitis and GERD that began follow-up time on September 11, 2006.

\begin{tabular}{|c|c|c|c|c|c|c|}
\hline \multirow[t]{2}{*}{ Estimate } & \multicolumn{2}{|c|}{$\begin{array}{l}\text { Model } 1 \text { - total effect } \\
\text { of WTC exposure, } \\
\text { OAD ignored }\end{array}$} & \multicolumn{2}{|c|}{$\begin{array}{c}\text { Model 2- effect of OAD } \\
\text { diagnosis, WTC exposure } \\
\text { ignored }\end{array}$} & \multicolumn{2}{|c|}{$\begin{array}{c}\text { Model 3-regression } \\
\text { direct effect of WTC } \\
\text { exposure }\end{array}$} \\
\hline & Relative rate & $95 \% \mathrm{Cl}$ & Relative rate & $95 \% \mathrm{Cl}$ & Relative rate & $95 \% \mathrm{Cl}$ \\
\hline \multicolumn{7}{|c|}{ Models for incidence of chronic rhinosinusitis } \\
\hline WTC exposure: high vs. low & 1.93 & $1.52-2.44$ & $\mathrm{~N} / \mathrm{A}$ & & 1.74 & $1.38-2.20$ \\
\hline WTC exposure: high vs. moderate & 1.26 & $1.09-1.46$ & $\mathrm{~N} / \mathrm{A}$ & & 1.21 & $1.04-1.39$ \\
\hline WTC exposure: moderate vs. low & 1.52 & $1.24-1.88$ & $\mathrm{~N} / \mathrm{A}$ & & 1.45 & $1.17-1.78$ \\
\hline Effect of OAD diagnosis & $\mathrm{N} / \mathrm{A}$ & & 3.52 & $3.07-4.04$ & 3.45 & $3.00-3.96$ \\
\hline \multicolumn{7}{|c|}{ Models for incidence of gastroesophageal reflux disease } \\
\hline WTC exposure: high vs. low & 1.40 & $1.20-1.64$ & $\mathrm{~N} / \mathrm{A}$ & & 1.25 & $1.07-1.47$ \\
\hline WTC exposure: high vs. moderate & 1.16 & $1.04-1.29$ & $N / A$ & & 1.10 & $0.99-1.23$ \\
\hline WTC exposure: moderate vs. low & 1.21 & $1.06-1.39$ & $\mathrm{~N} / \mathrm{A}$ & & 1.14 & $0.99-1.30$ \\
\hline Effect of OAD diagnosis & $\mathrm{N} / \mathrm{A}$ & & 2.79 & $2.53-3.07$ & 2.75 & $2.50-3.03$ \\
\hline
\end{tabular}

All models were fit using piecewise exponential survival models and included age, retirement status, smoking status, and season as covariates. Models are defined in the text in Eqs 1-3.

follow-up may be evidence for a non-biologic cause early in the follow-up period.

We also acknowledge that reverse causality of the aerodigestive conditions is biologically plausible: that is, CRS or GERD may trigger OAD symptoms such as cough and shortness of breath. If therapeutic interventions intended to improve OAD symptoms were ineffective because the symptoms were due to CRS or GERD, further diagnostic testing could have confirmed CRS or GERD as the etiology of cough and shortness of breath. A main limitation of this study is that the low incidence rates of CRS and GERD in the first 5 years after 9/11 did not allow us to statistically test this hypothesis. Further, the date of diagnosis may not coincide with disease onset if participants delay seeking treatment, which complicates efforts to establish the temporal occurrence, and thus the causal relationship, of OAD, CRS, and GERD. Another limitation is we cannot determine whether individuals with CRS or GERD symptoms sought treatment outside of FDNY-BHS in the immediate years after 9/11, which could have partially accounted for the low incidence rates. Moreover, we observed that incidence rates of CRS, GERD, and OAD all increased after 2007, the year that FDNY WTC Health Program started to provide free medications for WTC-related health conditions. This further complicates our understanding of whether the increased incidence rates after 2007 reflect delayed diagnoses of CRS or GERD or the progressive development of disease. Finally, we acknowledge that there may be unmeasured 
TABLE 5 | Results from causal mediation analyses examining the effect of OAD diagnosis as a mediator for chronic rhinosinusitis and gastroesophageal reflux disease for follow-up beginning September 11, 2006.

\begin{tabular}{|c|c|c|c|c|c|c|}
\hline & \multicolumn{3}{|c|}{ High vs. low exposure } & \multicolumn{3}{|c|}{ Moderate vs. low exposure } \\
\hline & Estimate & & & Estimate & & \\
\hline \multicolumn{7}{|c|}{ Causal mediation models for chronic rhinosinusitis diagnosis } \\
\hline Controlled direct effect & 0.542 & 0.288 & 0.856 & 0.352 & 0.125 & 0.637 \\
\hline Interaction in absence of mediator & 0.001 & -0.017 & 0.013 & 0.000 & -0.007 & 0.005 \\
\hline Mediated interaction & 0.001 & -0.018 & 0.018 & 0.001 & -0.012 & 0.013 \\
\hline Pure indirect effect & 0.029 & 0.014 & 0.046 & 0.015 & 0.008 & 0.028 \\
\hline Total effect & 0.573 & 0.333 & 0.886 & 0.369 & 0.148 & 0.642 \\
\hline Proportion mediated & 0.057 & 0.026 & 0.108 & 0.048 & 0.021 & 0.101 \\
\hline \multicolumn{7}{|c|}{ Results from conventional models for chronic rhinosinusitis diagnosis } \\
\hline Regression direct effect & 0.554 & 0.322 & 0.788 & 0.372 & 0.157 & 0.577 \\
\hline Total regression effect & 0.658 & 0.419 & 0.892 & 0.419 & 0.215 & 0.631 \\
\hline \multicolumn{7}{|c|}{ Causal mediation models for gastroesophageal reflux disease } \\
\hline Controlled direct effect & 0.244 & 0.069 & 0.440 & 0.135 & -0.011 & 0.295 \\
\hline Interaction in absence of mediator & -0.002 & -0.016 & 0.010 & 0.000 & -0.007 & 0.006 \\
\hline Mediated interaction & -0.002 & -0.014 & 0.012 & 0.000 & -0.011 & 0.012 \\
\hline Pure indirect effect & 0.034 & 0.018 & 0.054 & 0.018 & 0.008 & 0.030 \\
\hline Total effect & 0.275 & 0.103 & 0.464 & 0.152 & 0.012 & 0.305 \\
\hline Proportion mediated & 0.136 & 0.056 & 0.332 & 0.289 & 0.038 & 0.696 \\
\hline \multicolumn{7}{|c|}{ Results from conventional models for gastroesophageal reflux disease diagnosis } \\
\hline Regression direct effect & 0.223 & 0.068 & 0.385 & 0.131 & -0.010 & 0.262 \\
\hline Total regression effect & 0.336 & 0.182 & 0.495 & 0.191 & 0.058 & 0.329 \\
\hline
\end{tabular}

Causal mediation models are defined in Eqs 5 and 6 in the text; conventional models are defined in Eqs 1 and 3. The quantities shown in the tables are defined in Eqs 7 and 4 in the text. Confidence intervals for causal mediation models based on 1000 bootstrap simulations; confidence intervals for conventional models based are large sample Wald-type intervals.

confounders in this study, which may bias the results of mediation analysis in log-linear models. Although we have included major risk factors for both CRS and GERD as confounders in the respective models, there may be other confounders that we have not captured.

A primary strength of this prospective cohort study is our access to FDNY's electronic medical records database, which includes dates of diagnoses documented before and after 9/11. We were able to exclude individuals with pre-9/11 evidence of any of three aerodigestive conditions, so we are confident that the diagnoses we included were new post-9/11. Diagnosis dates also allowed us to establish the temporal order of the exposure (WTC-exposure), posited mediator (OAD), and both health outcomes (CRS and GERD), enabling us to conduct mediation analyses.

The combined effects of upper and lower airways disease and GERD have resulted in persistent adverse symptoms, which continue to have a negative impact on the quality of life $(11,12,17,18)$. Study findings support continued medical monitoring of WTC-exposed individuals as well as long-term

\section{REFERENCES}

1. Lioy PJ, Weisel CP, Millette JR, Eisenreich S, Vallero D, Offenberg J, et al. Characterization of the dust/smoke aerosol that settled east of the World Trade Center (WTC) in lower Manhattan after the collapse of the WTC 11 September 2001. Environ Health Perspect (2002) 110(7):703-14. doi:10.1289/ ehp. 02110703

2. Hall CB, Liu X, Zeig-Owens R, Webber MP, Aldrich TK, Weakley J, et al. The duration of an exposure response gradient between incident obstructive airways disease and work at the World Trade Center site: 2001-2011. PLoS Curr monitoring and treatment for future disasters with highintensity exposures.

\section{AUTHOR CONTRIBUTIONS}

$\mathrm{CH}$ conceptualized the study and wrote the first draft of the manuscript with critical revisions from JY, RZ-O, MPW, MW, and DP. XL, JY, RZ-O, JW, and TS contributed to data analysis and data management. All authors read and approved the final manuscript and agree to be accountable for all aspects of the work.

\section{FUNDING}

This research was supported by contracts and cooperative agreements from the United States National Institute of Occupational Safety and Health, U01 OH010412, and U01 OH010711, principal investigator Charles B. Hall for both; U01 OH010726 and U01 $\mathrm{OH} 011302$, principal investigator Michael D. Weiden for both; 200-2011-39378 principal investigator David J. Prezant; and 2002011-39383 principal investigator Kerry J. Kelly.

Disasters (2015) 7. doi:10.1371/currents.dis.8a93e7682624698558a76a1fa $8 \mathrm{c} 5893 \mathrm{f}$

3. Weakley J, Hall CB, Liu X, Zeig-Owens R, Webber MP, Schwartz T, et al. The effect of World Trade Center exposure on the latency of chronic rhinosinusitis diagnoses in New York City firefighters: 2001-2011. Occup Environ Med (2016) 73(4):280-3. doi:10.1136/oemed-2015-103094

4. Brackbill RM, Hadler JL, DiGrande L, Ekenga CC, Farfel MR, Friedman S, et al. Asthma and posttraumatic stress symptoms 5 to 6 years following exposure to the World Trade Center terrorist attack. JAMA (2009) 302(5):502-16. doi:10.1001/jama.2009.1121 
5. Li J, Brackbill RM, Stellman SD, Farfel MR, Miller-Archie SA, Friedman $S$, et al. Gastroesophageal reflux symptoms and comorbid asthma and posttraumatic stress disorder following the 9/11 terrorist attacks on World Trade Center in New York City. Am J Gastroenterol (2011) 106(11):1933-41. doi:10.1038/ajg.2011.300

6. Kwon S, Putman B, Weakley J, Hall CB, Zeig-Owens R, Schwartz T, et al. Blood eosinophils and World Trade Center exposure predict surgery in chronic rhinosinusitis. A 13.5-year longitudinal study. Ann Am Thorac Soc (2016) 13(8):1253-61. doi:10.1513/AnnalsATS.201511-742OC

7. Sayuk GS, Drossman DA. Gastroesophageal reflux symptoms in 9/11 survivors and workers: insights gained from tragic losses. Am J Gastroenterol (2011) 106(11):1942-5. doi:10.1038/ajg.2011.357

8. Yip J, Webber MP, Zeig-Owens R, Vossbrinck M, Singh A, Kelly K, et al. FDNY and 9/11: clinical services and health outcomes in World Trade Center-exposed firefighters and EMS workers from 2001 to 2016. Am J Ind Med (2016) 59(9):695-708. doi:10.1002/ajim.22631

9. Prezant D, Kelly K, Jackson B, Peterson D, Feldman D, Baron S, et al. In: Centers for Disease Control and Prevention, editor. Use of Respiratory Protection among Responders at the World Trade Center Site - New York City, September 2001. Atlanta: MMWR Morb Mortal Wkly Rep (2002). p. 6-8.

10. de la Hoz RE, Shohet MR, Chasan R, Bienenfeld LA, Afilaka AA, Levin SM, et al. Occupational toxicant inhalation injury: the World Trade Center (WTC) experience. Int Arch Occup Environ Health (2008) 81(4):479-85. doi:10.1007/s00420-007-0240-x

11. Wisnivesky JP, Teitelbaum SL, Todd AC, Boffetta P, Crane M, Crowley L, et al. Persistence of multiple illnesses in World Trade Center rescue and recovery workers: a cohort study. Lancet (2011) 378(9794):888-97. doi:10.1016/ s0140-6736(11)61180-x

12. Yip J, Zeig-Owens R, Webber MP, Kablanian A, Hall CB, Vossbrinck M, et al. World Trade Center-related physical and mental health burden among New York City Fire Department emergency medical service workers. Occup Environ Med (2016) 73(1):13-20. doi:10.1136/oemed-2014-102601

13. Prezant DJ, Levin S, Kelly KJ, Aldrich TK. Upper and lower respiratory diseases after occupational and environmental disasters. Mt Sinai J Med (2008) 75(2):89-100. doi:10.1002/msj.20028
14. Prezant DJ, Weiden M, Banauch GI, McGuinness G, Rom WN, Aldrich $\mathrm{TK}$, et al. Cough and bronchial responsiveness in firefighters at the World Trade Center site. N Engl J Med (2002) 347(11):806-15. doi:10.1056/ NEJMoa021300

15. Glaser MS, Webber MP, Zeig-Owens R, Weakley J, Liu X, Ye F, et al. Estimating the time interval between exposure to the World Trade Center disaster and incident diagnoses of obstructive airway disease. Am J Epidemiol (2014) 180(3):272-9. doi:10.1093/aje/kwu137

16. VanderWeele TJ. Explanation in Causal Inference: Methods for Mediation and Interaction. USA: Oxford University Press (2015).

17. Weakley J, Webber MP, Gustave J, Kelly K, Cohen HW, Hall CB, et al. Trends in respiratory diagnoses and symptoms of firefighters exposed to the World Trade Center disaster: 2005-2010. Prev Med (2011) 53(6):364-9. doi:10.1016/j.ypmed.2011.09.001

18. Webber MP, Glaser MS, Weakley J, Soo J, Ye F, Zeig-Owens R, et al. Physician-diagnosed respiratory conditions and mental health symptoms 7-9 years following the World Trade Center disaster. Am J Ind Med (2011) 54(9):661-71. doi:10.1002/ajim.20993

Conflict of Interest Statement: The authors declare that the research was conducted in the absence of any commercial or financial relationships that could be construed as a potential conflict of interest.

The reviewer SDM and handling Editor declared their shared affiliation, and the handling Editor states that the process nevertheless met the standards of a fair and objective review.

Copyright (c) 2017 Liu, Yip, Zeig-Owens, Weakley, Webber, Schwartz, Prezant, Weiden and Hall. This is an open-access article distributed under the terms of the Creative Commons Attribution License (CC BY). The use, distribution or reproduction in other forums is permitted, provided the original author (s) or licensor are credited and that the original publication in this journal is cited, in accordance with accepted academic practice. No use, distribution or reproduction is permitted which does not comply with these terms. 